\title{
EI DESMANTELAMIENTO DE PROPIEDADES, PLANTA Y EQUIPO: CASO DE ANÁLISIS DEL SISTEMA ELÉCTRICO DEL CANTÓN LA TRONCAL DE PROPIEDAD DE LA CNEL EP REGIONAL MILAGRO Y DE LA CENTROSUR C.A.
}

\author{
DISMANTLING OF PROPERTY, PLANT AND EQUIPMENT \\ IN THE ELECTRICAL SYSTEM ON TRONCAL CITY (ECUADOR), PROPERTY OF "CNEL EP \\ REGIONAL MILAGRO AND CENTROSUR C.A"
}

Recibido: 17/09/2017

Aceptado: 19/12/2017

\author{
Gladys Victoria Fernández Avilés ${ }^{1}$ \\ econgladysfernandeza@gmail.com \\ gladys.fernandez@ucuenca.edu.ec
}

\section{Resumen}

El presente artículo tiene como objetivo aplicar las NIC 16 y 37, respecto al tratamiento del desmantelamiento de Propiedades, Planta y Equipo en el sistema eléctrico del Cantón la Troncal de propiedad de la CNEL EP Regional Milagro y de la CENTROSUR C.A.; a través de una propuesta metodológica, para su incorporación en los registros contables y preparación de estados financieros acordes a la normativa vigente, que permitan tomar decisiones oportunas.

Palabras clave: Desmantelamiento, Restauración, Normas Internacionales de Información Financiera, NIIF-, Normas Internacionales de Contabilidad -NIC-, Valor presente, Vida útil.

\section{Abstract}

The aim of this paper is to apply International Accounting Standards 16 and 37, regarding to treatment of the dismantling of property, plant and equipment in the electrical system on Troncal City (Ecuador), property of "CNEL EP Regional Milagro and CENTROSUR C.A"; through a methodological proposal, for its incorporation into the accounting records and the making of financial statements in accordance with current regulations, which allow timely decisions.

Keywords: Dismantling, Restoration, International Financial Reporting Standards -IFRS International Accounting Standards -IAS-, Present Value, Useful Life.

\footnotetext{
Directora de la Carrera de Contabilidad y Auditoría y Docente Titular de la Facultad de Ciencias Económicas y Administrativas de la Universidad de Cuenca. Directora de la Regional 2 de la Contraloría General del Estado, Auditora Gubernamental en varias entidades y empresas públicas del Cantón Cuenca.

ORCID: 0000-0002-8448-0640
} 


\section{Introducción}

El objetivo del presente artículo es aplicar las NIC y NIIF, respecto al tratamiento del desmantelamiento de Propiedades, Planta y Equipo. El trabajo cobra importancia en razón de que no se aplica el desmantelamiento en los registros contables de las empresas privadas y públicas en general, siendo la Centrosur C.A., pionera en incluir en el tratamiento contable y financiero, el tema del desmantelamiento, para lo cual se desarrolla una metodología para su aplicación.

Las Normas Internacionales de Contabilidad (NIC), son las primeras normas emitidas por el organismo internacional IASB (International Accounting Standard Board), que se encuentran en permanente revisión y cambio para adaptarse a las necesidades de los usuarios de la información financiera; y, en el año de 1996 la Federación Nacional de Contadores del Ecuador comenzó a formar parte de la Federación Internacional de Contadores (IFAC) y adoptó las NIC. En el año 2011 la Superintendencia de Compañías de Ecuador remitió el Reglamento para la aplicación de las Normas Internacionales de Información Financiera (NIIF) completas y para PYMES.

Todas las empresas del país tienen que utilizar el mismo marco normativo y contable, que permita la comparación de la información financiera, a través de la aplicación de las $\mathrm{NIC}^{2}$ y las NIIF; las mismas que se basan en principios y no en reglas, contribuyendo al mejoramiento de la calidad de los estados financieros.

Tratar el desmantelamiento de Propiedades, Planta y Equipo, implica entender lo que la NIC 16 establece respecto al tema, como son los objetivos, alcance, los principios de reconocimiento, la capitalización de los costos con respecto al reconocimiento inicial, cuáles son los costos directamente atribuibles, los costos no capitalizables, métodos para adquirir los activos, sus componentes, cuál es el valor del desmantelamiento que se considera como costo de Propiedades, Planta y Equipo, qué efecto tiene el desmantelamiento en los inventarios. Se aplica para todos los activos, generan impuestos fiscales, entre otros.

La NIC 16, establece que, al costo de un elemento fijo tangible, se debe incrementar, por la estimación inicial de los costos de desmantelamiento o retiro del activo y la restauración del lugar donde está situado cuando constituyen obligaciones para la empresa. La NIC 37, se refiere a que la estimación inicial será constituida a valor presente y contiene requerimientos sobre la forma a valorar los pasivos (provisiones) por desmantelamiento, retiro o restauración; señalando, entre otros aspectos, que la actualización de la provisión constituye un gasto financiero.

El término desmantelamiento hace referencia al desalojo, destrucción o reubicación de un activo, evento que puede darse en el momento de la adquisición del mismo y que se va a realizar en el futuro. Dicho evento no es para todos los activos sino para aquellos en los cuales se determina que cuando se termina su vida útil se debe necesariamente movilizarlos, destruirlos o reubicarlos; siendo importante definir el tiempo de utilización del activo, la vida útil y la estimación inicial del valor actual a consecuencia del desmantelamiento o retiro del activo, que serán los costos de rehabilitación del lugar sobre el que se asienta el activo.

El retiro de los activos del Sistema Eléctrico de la CNEL EP (Empresa Eléctrica Pública Estratégica Corporación Nacional de Electricidad) regional Milagro y de la CENTROSUR C.A. (Empresa Eléctrica Regional Centro Sur), conlleva desembolsos adicionales necesarios para cada etapa funcional; así por ejemplo, para Edificios y Estructuras; Equipo de Subestación; Postes, Torres y Accesorios Línea de S/T; Conductores y Accesorios en Línea de S/T; Postes Torres y Accesorios en M.T y B.T; Conductores y Accesorios en MT y BT; Transformadores de Distribución; Sistema de Alumbrado Público; Acometidas en Sistemas de Medición y Medidores de Energía.

2 Particularmente las Normas 16 y 37. 


\section{2.- Marco teórico}

Pese a que las NIC y NIIF son de aplicación obligatoria en el país, la mayoría de empresas públicas y privadas, obligadas a aplicar dicha normativa, no evidencian su aplicación; particularmente las NIC 16 y 37 , debido entre otras causas, a la ausencia de un proceso metodológico claramente definido para su registro, razón por la cual, la bibliografía disponible se encuentra únicamente en procesos de aproximación a nivel de trabajos de titulación tanto de pregrado y de posgrado en varias universidades del país; y, el marco teórico para estos trabajos es precisamente las NIC y NIIF.

En base a lo anotado, el marco teórico utilizado en esta investigación es únicamente lo dispuesto en las NIC 16 y 37.

\subsection{Norma Internacional de Contabilidad 16 - Propiedades, Planta y Equipo -}

\subsubsection{Objetivo de la Norma}

El objetivo de esta Norma es "(...) prescribir el tratamiento contable de Propiedades, Planta y Equipo, de forma que los usuarios de los estados financieros puedan conocer la información acerca de la inversión que la entidad tiene en sus propiedades, planta y equipo, así como los cambios que se hayan producido en dicha inversión. Los principales problemas que presenta el reconocimiento contable de propiedades, planta y equipo son la contabilización de los activos, la determinación de su importe en libros y los cargos por depreciación y pérdidas por deterioro que deben reconocerse con relación a los mismos...". (IFRS Fundation, 2014).

\subsubsection{Valoración del activo en el momento del reconocimiento inicial}

Todo elemento de Propiedades, Planta y Equipo (activos tangibles), debe cumplir ciertas condiciones para ser reconocido como un activo y se medirá por su costo; y sus componentes son:

“... (a) Su precio de adquisición, incluidos los aranceles de importación y los impuestos indirectos no recuperables que recaigan sobre la adquisición, después de deducir cualquier descuento o rebaja del precio.

(b) Todos los costos directamente atribuibles a la ubicación del activo en el lugar y en las condiciones necesarias para que pueda operar de la forma prevista por la gerencia (...)".

Estos costos pueden incluir: los costos de preparación del emplazamiento, los costos de entrega y manipulación inicial, los de instalación y montaje, y los de comprobación de que el activo funciona adecuadamente.

(c) “(...) La estimación inicial de los costos de desmantelamiento o retiro del elemento, así como la rehabilitación del lugar sobre el que se asienta, la obligación en que incurre la entidad cuando adquiere el elemento o como consecuencia de haber utilizado dicho elemento durante un determinado período, con propósitos distintos al de producción de inventarios durante tal periodo...”. El subrayado es propio. (IFRS Fundation, 2014).

\subsubsection{Principios de reconocimiento del activo}

Se reconoce como Propiedades, Planta y Equipo únicamente cuando:

“(...) a.- Sea probable que la empresa obtenga los beneficios económicos futuros derivados del mismo; y, 
b.- El costo del activo para la empresa puede medirse con fiabilidad (...)". (IFRS Fundation, 2017).

\subsubsection{Medición posterior al reconocimiento de Propiedades, Planta y Equipo}

Las entidades elegirán como política contable el modelo del costo o el modelo de revaluación.

1.- Modelo del costo, donde las Propiedades, Planta y Equipo es igual al costo menos la depreciación acumulada y el valor acumulado de las pérdidas por deterioro del valor;

2.- Modelo de revaluación, las Propiedades, Planta y Equipo es llevado al valor revaluado, que es el valor razonable, menos la depreciación acumulada y el importe acumulado de las pérdidas por deterioro. Las revaluaciones se harán con regularidad para asegurar que el valor en libros refleje un valor razonable y en el caso de activos que tengan cambios significativos y volátiles en su valor razonable, necesitan revaluaciones anuales. Las revaluaciones frecuentes no serán necesarias para Propiedades, Planta y Equipo con variaciones insignificantes en su valor razonable, en estos casos son suficientes las revaluaciones hechas cada tres o cinco años....". (IFRS Fundation, 2017).

\subsubsection{Definición de términos:}

- Costo: es el importe de efectivo o equivalentes al efectivo pagados, o el valor razonable de la contraprestación entregada, para adquirir un activo en el momento de su adquisición o construcción.

- Depreciación: es la distribución sistemática del importe depreciable de un activo a lo largo de su vida útil.

- Importe depreciable: es el costo de un activo, o el importe que lo haya sustituido, menos su valor residual.

- Importe en libros: es el valor por el que se reconoce un activo, una vez deducida la depreciación acumulada y las pérdidas por deterioro del valor acumulado.

- Importe recuperable: es el mayor entre el valor razonable menos los costos de venta de un activo y su valor de uso.

- Propiedades, Planta y Equipos son los activos tangibles que:

a. Posee una entidad para su uso en la producción o suministro de bienes y servicios, para arrendarlos a terceros o para propósitos administrativos; y,

b. Se espera utilizar durante más de un periodo.

- Valor residual de un activo: es el valor estimado que la empresa podría obtener por la enajenación o disposición de un activo, después de deducir los costos estimados para su enajenación o disposición, si el activo ya hubiera alcanzado la edad y las demás condiciones esperadas al término de su vida útil.

- Vida útil: es el período durante el cual se espera utilizar el activo depreciable por parte de la empresa; o el número de unidades de producción o similares que se espera obtener del mismo por parte de la empresa..." (IFRS Fundation, 2017).

\subsection{Norma Internacional de Contabilidad 37: Provisiones, Pasivos Contingentes y Activos Contingentes}

\subsubsection{Objetivo de la Norma}


El objetivo de esta Norma es “(...) asegurar que se utilicen las bases apropiadas para el reconocimiento y la medición de las provisiones, pasivos contingentes y activos contingentes, así como que se revele la información complementaria suficiente, por medio de las notas, como para permitir a los usuarios comprender la naturaleza, calendario de vencimiento e importes, de las anteriores partidas...". El subrayado es propio. (IFRS Fundation, 2017).

\subsubsection{Alcance de la Norma}

Esta Norma debe ser aplicada por todas las entidades al proceder a contabilizar sus provisiones, pasivos contingentes y activos contingentes. En esta Norma se define las provisiones como pasivos de cuantía o vencimiento inciertos.

\subsubsection{Reconocimiento de las provisiones}

Una provisión debe reconocerse cuando se den las siguientes condiciones:

a.- Una entidad tiene una obligación presente (ya sea legal o implícita) como resultado de un suceso pasado.

b.- Es probable que la entidad tenga que desprenderse de recursos, que incorporen beneficios económicos para cancelar tal obligación; y

c.- Puede hacerse una estimación fiable del importe de la obligación.

De no cumplirse las tres condiciones indicadas, la empresa no debe reconocer la provisión.

\subsubsection{Definición de términos:}

- “Provisión: es un pasivo sobre el que existe incertidumbre acerca de su cuantía o vencimiento.

- Pasivo, es una obligación presente de la entidad, surgida a raíz de sucesos pasados, al vencimiento de la cual, y para cancelarla, la entidad espera desprenderse de recursos que incorporan beneficios económicos.

- $\quad$ El suceso que da origen a la obligación: es todo aquel suceso del que nace una obligación de pago, de tipo legal o implícita para la entidad, de forma que a la entidad no le queda otra alternativa más realista que satisfacer el importe correspondiente.

- Obligación Implícita: es la que se deriva de las actuaciones de la propia entidad, en las que:

a.- Debido a un patrón establecido de comportamiento en el pasado, a políticas empresariales que son de dominio público o a una declaración efectuada de forma suficientemente concreta, la entidad haya puesto de manifiesto ante terceros que está dispuesta a aceptar cierto tipo de responsabilidades; $y$

b.- Como consecuencia de lo anterior, la entidad haya creado una expectativa válida, ante aquellos terceros con los que debe cumplir sus compromisos o responsabilidades. El subrayado es propio. (IFRS Fundation, 2017 ).

- Pasivo Contingente:

a.- Una obligación posible, surgida a raíz de sucesos pasados y cuya existencia ha de ser confirmada sólo por la ocurrencia o la falta de ocurrencia de uno de más hechos futuros, sucesos inciertos que no están enteramente bajo el control de la entidad.

b.- Una obligación presente, surgida a raíz de sucesos pasados, que no se han reconocido contablemente. 
- Suceso pasado: el suceso pasado del que se deriva la obligación, se da cuando la entidad no tenga, como consecuencia del mismo, otra alternativa más realista que atender el pago de una obligación creada por tal suceso, tan solo en las siguientes condiciones:

a.- El pago de la obligación viene exigido por la ley; o

b.- Al tratarse de una obligación implícita, cuando el suceso (que puede ser una actuación de la propia entidad) haya creado una expectativa válida ante aquellos terceros con los que debe cumplir sus compromisos o responsabilidades.

Ejemplos de tales obligaciones son las multas medioambientales o los costos de reparación de los daños medioambientales causados en contra de la ley, puesto que, tanto en uno como en otro caso, y para pagar los compromisos correspondientes, se derivarán para la empresa salidas de recursos que incorporan beneficios económicos, con independencia de las actuaciones futuras que esta lleve a cabo.

- Riesgos e incertidumbres: para realizar la mejor estimación de la provisión, deben tomarse en cuenta los riesgos e incertidumbres que, inevitablemente, rodean a la mayoría de los sucesos y las circunstancias concurrentes a la valoración de la misma.

- Valor presente: cuando resulte importante el efecto financiero producido por el descuento, el importe de la provisión debe ser el valor presente de los desembolsos que se espera sean necesarios para cancelar la obligación. El importe de las provisiones, por tanto, será objeto de descuento cuando el efecto de hacerlo resulte significativo. (El subrayado es propio).

- Cambios en el valor de las provisiones: las provisiones deben ser objeto de revisión al final de cada periodo sobre el que se informa, y ajustadas consiguientemente para reflejar en cada momento la mejor estimación disponible. En el caso de que no sea ya probable la salida de recursos, que incorporen beneficios económicos, para cancelar la obligación correspondiente, se procederá a liquidar o revertir la provisión, así por ejemplo en el caso de activos que tengan cambios significativos y volátiles en su valor razonable, con revaluaciones anuales y las Propiedades, Planta y Equipo con variaciones insignificantes en su valor razonable, en estos casos son suficientes las revaluaciones hechas cada tres o cinco años.

- Aplicación de la provisiones: cada provisión deberá ser utilizada sólo para afrontar los desembolsos para los cuales fue originalmente reconocida...." (IFRS Fundation, 2017 ).

\section{Metodología}

Para la aplicación de la investigación en el tratamiento del desmantelamiento de Propiedades, Planta y Equipo en la CNEL EP Y CENTROSUR C.A., se inicia con la valoración de los activos, a través de un peritaje que valida los diferentes activos de las empresas ${ }^{3}$, y con los resultados obtenidos se aplica el proceso definido para el desmantelamiento, con la inserción contable de la aplicación metodológica propuesta, con efectos directos en los Estados Financieros.

La metodología que se aplica en esta investigación de tratamiento del desmantelamiento de Propiedades, Planta y Equipo: caso CNEL EP Y CENTROSUR C.A., es de carácter cualitativo, debido a que se enfoca a los aspectos contables, económicos y financieros de las empresas eléctricas definidas, lo que permite analizar el registro del costo por desmantelamiento y sus provisiones, no se va a centrar en el análisis de estados financieros y/o resultados contables; como soporte se basa en el análisis de conceptos básicos que permite desarrollar esta investigación a través del método deductivo que va de lo general a lo particular.

Entre las técnicas de recolección de datos de la investigación que se aplican en este trabajo, se encuentran: la observación, verificación física, análisis documental y la entrevista a los funcionarios responsables del departamento encargado del control y administración de Propiedades, Planta y Equipo de la CNEL EP y CENTROSUR C.A., en la aplicación de las NIC 16, 37 y NIIF.

Además, se complementa el análisis con el enfoque cuantitativo mediante el método de razonamiento

3 Peritaje realizado por el Arq. Jorge Rodríguez Jiménez y su equipo de trabajo conformado por: Econ. CPA Gladys Victoria Fernández Avilés Mgs e Ing. Com. Karla Argudo Tello. 
deductivo, que va de lo general a lo particular, se hace uso de una serie de herramientas e instrumentos que permitan conseguir los objetivos propuestos en la investigación, este método permite analizar las variables planteadas, bajo una hipótesis explicativa que tiene como fundamento la prueba de la hipótesis y busca que las conclusiones lleven a la formulación o el contraste de leyes o principios científicos.

\subsection{Registro contable y cálculo del desmantelamiento}

El proceso seguido para el registro contable de los activos del Sistema Eléctrico del Cantón la Troncal de propiedad de la CNEL EP Regional Milagro y de la CENTROSUR C.A es el siguiente:

1.- Se registra el costo del activo según las etapas funcionales por su adquisición o construcción, ya sea al contado o a crédito; al momento constituye los valores históricos que constan en el registro contable de la CNEL EP Regional Milagro y de la CENTROSUR C.A.

Tabla 1.

\begin{tabular}{|l|c|c|}
\hline \multicolumn{1}{|c|}{ Descripción } & Debe & Haber \\
\hline Costo del activo & xxxxx & \\
\hline Bancos & & xxxxx \\
\hline Por la compra o construcción del activo. & & \\
\hline
\end{tabular}

Elaboración: La autora

2.- Se contabiliza el costo por desmantelamiento, valor que incrementa el costo del activo, mediante el cálculo del valor presente y con crédito a una cuenta de provisión con el nombre del activo que lo identifica; registro que se debe realizar en la fecha en la cual se calcula el valor por desmantelamiento.

El cálculo de la provisión, se realiza con la fórmula del valor presente que sirve para determinar el valor de la inversión actual. El valor presente (VP) de un capital (M), que se recibirá al final de un tiempo (n) a una tasa de interés (i), se describe a continuación:

\section{Donde:}
$\mathrm{VP}=$ Valor presente
$\mathrm{M}=\quad$ Valor histórico del activo
$\mathrm{i}=\quad$ Tasa de interés Activa Referencial emitida por el Banco Central del Ecuador, vigente a junio de 2015. (8,70\%)
$\mathrm{n}=\quad$ Vida útil del activo.

\begin{tabular}{|l|c|c|}
\hline \multicolumn{1}{|c|}{ Descripción } & Debe & Haber \\
\hline Costo por desmantelamiento del activo & xxxx & \\
\hline Provisión por desmantelamiento del activo & & xxxx \\
\hline Incremento del activo según NIC 16 por desmantelamiento. & \\
\hline
\end{tabular}

3.- Se contabiliza el gasto por depreciación del costo histórico o inicial del activo, calculado de acuerdo al método de depreciación aplicado. 


\begin{tabular}{|c|c|c|}
\hline \multicolumn{1}{|c|}{ Descripción } & Debe & Haber \\
\hline Gasto por depreciación del activo & $\operatorname{xxxxx}$ & \\
\hline \multicolumn{2}{|c|}{ Depreciación acumulada del activo } & \\
\hline \multicolumn{2}{|c|}{ Por el registro de la depreciación del valor histórico del activo. } \\
\hline
\end{tabular}

4.- Se procede a registrar contablemente el gasto por la provisión del valor por desmantelamiento calculado, que se incrementó al costo del activo y acreditando a una cuenta de provisión acumulada por desmantelamiento, de acuerdo al cálculo del valor presente durante toda la vida útil del activo y con la tasa de interés activa establecida por el Banco Central del Ecuador.

\begin{tabular}{|l|c|c|}
\hline \multicolumn{1}{|c|}{ Descripción } & Debe & Haber \\
\hline Gasto por provisión del desmantelamiento del activo & xxxxx & \\
\hline Provisión acumulada por desmantelamiento del activo & & xxxxx \\
\hline \multicolumn{2}{l|}{ Registro del valor de la provisión por el costo del activo por desmantelamiento, } \\
calculado de acuerdo al método de depreciación aplicado previamente. \\
\hline
\end{tabular}

5.- Al término de cada año fiscal, se contabiliza la actualización de la provisión como gastos financieros por desmantelamiento y esto por cada año hasta completar la vida útil del activo.

\begin{tabular}{|l|c|c|}
\hline \multicolumn{1}{|c|}{ Descripción } & Debe & Haber \\
\hline Gasto financiero por desmantelamiento del activo & $\mathrm{xxxxx}$ & \\
\hline \multicolumn{2}{|c|}{ Provisión por desmantelamiento del activo } & $\mathrm{xxxxx}$ \\
\hline $\begin{array}{l}\text { Registro del valor calculado de acuerdo al valor presente del activo por } \\
\text { desmantelamiento durante toda la vida del activo. }\end{array}$ \\
\hline
\end{tabular}

Una vez identificado el importe estimado de los costos por desmantelamiento o retiro del activo inmovilizado, así como la rehabilitación del lugar sobre el que se asienta los activos, la empresa adquiere estas obligaciones y se requiere el registro contable respectivo, del incremento del costo del activo, la provisión y gastos financieros producto del desmantelamiento, como se detalló previamente.

\section{Discusión y resultados}

\section{Características del manejo de las Propiedades, Planta y Equipo: del sistema eléctrico del Cantón la} Troncal de propiedad de la CNEL EP regional Milagro y de la CENTROSUR C.A.

\subsection{Los costos por desmantelamiento incrementan el valor de los activos}

La obligación de incluir el valor actual de los gastos de desmantelamiento como mayor valor del inmovilizado, supone en principio una ruptura de la concepción tradicional del precio de adquisición, entendiendo por este el importe pagado.

No obstante, si se piensa en términos de flujo de caja, cuando se realiza una inversión, esta producirá entradas de efectivo durante toda su vida útil, pero, ¿qué costos tiene esos flujos de caja? Pues por la inversión inicial una salida en el momento de la puesta en marcha y otra salida de efectivo en el momento del desmantelamiento. Por lo tanto, el valor del inmovilizado incluirá el costo actualizado de todos los pagos 
de la inversión necesaria para la obtención de los ingresos, se produzcan los pagos ahora o al final de la vida útil del bien.

La incorporación de este componente del costo a la valoración inicial del activo se producirá en la fecha en la que la empresa incurra en la obligación, que puede ser al momento de la adquisición o de la construcción del activo; o en cualquier época de la vida útil del mismo en la que se reconoce la provisión por desmantelamiento.

Por su parte, la valoración inicial de Propiedades, Planta y Equipo podrá verse alterada por cambios en estimaciones contables que modifiquen el importe de la provisión asociada a los costos de desmantelamiento en más o en menos.

\subsection{Provisiones por desmantelamiento y retiro}

El valor de la provisión será la mejor estimación del desembolso que la empresa debe efectuar para liquidar la obligación, lo que exige determinar la provisión por el valor presente de la obligación.

La provisión por desmantelamiento o retiro no es más que el valor estimado de los costos de desmantelamiento o retiro del inmovilizado, así como la rehabilitación del lugar sobre el que se asienta. La empresa puede incurrir en estas obligaciones en el momento de adquirir dicho bien o para poder utilizar el mismo durante un determinado período de tiempo.

De esta manera la NIC 37 trata específicamente el tema de la "Provisión por Desmantelamiento", la misma que refleja en la contabilidad el valor estimado de los costos por desmantelamiento o retiro del activo fijo en una empresa, así como la rehabilitación del lugar sobre el que se asienta dicho activo.

La estimación inicial será constituida a valor presente y contiene requerimientos sobre la forma a valorar los pasivos (provisiones) por desmantelamiento, retiro o restauración; señalando, entre otros aspectos, que la actualización de la provisión constituye un gasto financiero.

Por lo tanto, el importe estimado del valor actual de los costos del desmantelamiento o retiro se registrarán contablemente como provisión, con cargo a la correspondiente cuenta de Propiedades, Planta y Equipo, cuando se incurra en la obligación, bien en el momento de su adquisición o posteriormente.

La provisión no figurará como un gasto total, como se determina en otras provisiones, sino que incrementará por una parte el valor de la inversión del bien y será consumida vía depreciación de las Propiedades, Planta y Equipo, ya que este activo incluirá el valor actualizado de la provisión.

Con el transcurso de los años se podrá ir ajustando el importe de la provisión por los incrementos o disminuciones originados por una nueva estimación, con cargo o abono, respectivamente, a la cuenta de Propiedad, Planta y Equipo.

Por otro lado, el importe de los ajustes que surjan por la actualización financiera de su valor, se tendrá que incrementar a la provisión, pero esta con cargo a la cuenta de gastos financieros por consecuencia de la actualización de la provisión.

\subsection{Las Propiedades, Planta y Equipo del sistema eléctrico del Cantón la Troncal: de propiedad de la CNEL EP regional Milagro y de la CENTROSUR C.A.}

El análisis y cálculo del desmantelamiento y la provisión se realizó para el SISTEMA ELÉCTRICO DEL CANTÓN LA TRONCAL DE PROPIEDAD DE LA CNEL EP REGIONAL MILAGRO Y DE LA CENTROSUR C.A., actualmente todos los activos forman parte de la CENTROSUR C.A.

Luego de varios análisis realizados al comportamiento de las diferentes etapas funcionales de los activos del Sistema Eléctrico del Cantón la Troncal de propiedad de la CNEL EP Regional Milagro y de la CENTROSUR CA; referente a la estimación del valor para desmantelamiento, se consideraron algunos parámetros tales como: el estado de conservación del inmovilizado material, la vida útil de los activos, el mantenimiento preventivo y correctivo implementado, la antigüedad del activo, servicio a los usuarios, 
crecimiento sostenible del sector eléctrico, incremento de proyectos hidroeléctricos, mayor demanda eléctrica en todos los sectores del país, desarrollo socioeconómico de los sectores más distantes, situación geográfica y ubicación del sistema eléctrico, sucesos futuros que puedan afectar al valor necesario para cancelar una obligación originados por cambios en las disposiciones legales, estimación fiable para el cumplimiento de las obligaciones por desmantelamiento, entre otras.

Las fases de las diferentes etapas funcionales de los activos del Sistema Eléctrico de la CNEL EP y de la CENTROSUR C.A, son la construcción, operación y retiro; para las dos primeras etapas se han desarrollado procesos de control administrativos, financieros y económicos; sin embargo, para la etapa de retiro se requiere aplicar la NIC 16 y 37 , que tiene como finalidad estimar el costo de desmantelamiento a futuro, precisamente cuando termine la vida útil del activo y por lo tanto medir el impacto financieroeconómico. Incluso en términos normativos se conoce de las multas medioambientales, la necesidad de considerar el costo por reparación de los daños medioambientales, que para la empresa significa salida de recursos, con independencia de las actuaciones futuras que esta lleve a cabo.

La fase de Retiro o Desmontaje: son los trabajos de retiro parcial o total de los elementos, que conforman las diferentes etapas funcionales de los activos del Sistema Eléctrico del Cantón la Troncal de propiedad de la CNEL EP Regional Milagro y de la CENTROSUR C.A, una vez que las mismas estén fuera de servicio por tramos o en su totalidad, ya sea por causa natural o porque han terminado su vida útil, en esta fase se determinará la Provisión de Desmontaje. Las fases de retiro pueden ser las siguientes:

a.- Desarmado: comprende el retiro o desarmado de torres, cables, conductores y remoción de los cimientos.

b.- Derrocamiento y producción de escombros: es el retiro de las estructuras a través del derrocamiento de las obras civiles iniciales.

c.- Transporte de piezas y escombros: corresponde a la remoción, evacuación y traslado de escombros fuera del área hacia la disposición final.

d.- Disposición final de residuos y desechos: la disposición de los residuos y desechos generados durante el desmantelamiento y derrocamiento; su clasificación y disposición se realizará según su naturaleza con la finalidad de no afectar al ecosistema.

e.- Conformación de suelo y cobertura vegetal: rellenos de las cimentaciones y compactación de terreno, reforestación de áreas desbrozadas, entre otros.

Ante la ausencia de parámetros, estándares y una metodología establecida para estimar el costo del desmantelamiento de los activos, es importante definir una estimación, como el importe reconocido por provisión que debe ser la mejor estimación y es el valor que se reflejará en los estados financieros, de los recursos necesarios para cancelar la obligación presente. En ningún caso la estimación por desmantelamiento será sobrevalorada o mayor al costo del activo.

\subsection{Porcentajes de depreciación y vida útil de las Propiedades, Planta y Equipo del sistema eléctrico del Cantón la Troncal: de propiedad de la CNEL EP Regional Milagro y de la CENTROSUR C.A.}

La vida útil y los porcentajes de depreciación establecidos para los activos de la CNEL EP y de la CENTROSUR C.A, son los definidos por el INECEL (Instituto Ecuatoriano de Electrificación); y, por lo dispuesto en el numeral 6 del artículo 28 del Reglamento para la Aplicación de la Ley de Régimen Tributario Interno, LORTI, publicado en el Registro Oficial Suplemento 209 del 8 de junio de 2010, Decreto Ejecutivo No. 374; para cada una de las etapas funcionales de los activos que se aplican actualmente para el registro contable, en base a los mismos se ha calculado los valores por desmantelamiento, los gastos por intereses y provisión, de acuerdo a las diferentes etapas funcionales, que se detallan a continuación: 


\section{Tabla 1.}

\begin{tabular}{|c|l|c|}
\hline No. & \multicolumn{1}{|c|}{ Descripción } & \multicolumn{1}{|c|}{ Vida útil } \\
\hline 1 & Edificios y Estructuras Subestación la Troncal & 30 \\
\hline 2 & Equipos de Subestación La Troncal & 30 \\
\hline 3 & $\begin{array}{l}\text { Postes, Torres y Accesorios Línea de S/T S/E TRONCAL- S/E } \\
\text { EL TRIUNFO }\end{array}$ & 30 \\
\hline 5 & $\begin{array}{l}\text { Conductores y Accesorios en Línea de S/T S/E Troncal - S/E El } \\
\text { Triunfo. }\end{array}$ & 30 \\
\hline 6 & Postes, Torres y Accesorios en M.T. y B.T. La Troncal & 25 \\
\hline 7 & Transformadores de Distribución La Troncal & 25 \\
\hline 9 & Sistema de Alumbrado Público La Troncal & 15 \\
\hline 10 & Medidores de Energía La Troncal & 15 \\
\hline
\end{tabular}

Fuente: Instituto Nacional de Electrificación

Elaboración: La autora

\subsection{Impacto financiero - económico}

Los activos del Sistema Eléctrico de la CNEL EP y de la CENTROSUR C.A, no registran contablemente lo dispuesto por las Normas Internacionales de Contabilidad 16 y 37, particularmente en lo que se refiere a los valores para el desmantelamiento, afectando a las cuentas de activos, pasivos, patrimonio, gastos y resultados.

El valor total del desmantelamiento del Sistema Eléctrico del Cantón la Troncal de propiedad de la CNEL EP Regional Milagro asciende a $1334.550,68$ USD, que representa el 15,84 \%, en promedio, con respecto al valor total de los activos históricos, dicho porcentaje difiere para cada una de las etapas funcionales de acuerdo a la vida útil de los activos; para aquellos que tienen una vida útil de 30 años el porcentaje del desmantelamiento es del 8,19\%, para los activos con una vida útil de 25 años es el $12,42 \%$ y para los activos que tienen una vida útil de 15 años es del 28,61\%; no obstante, en valores absolutos no son iguales, debido a que los valores históricos son diferentes, como se detalla en la Tabla 2.

Tabla 2. Resumen de valores totales por desmantelamiento de Propiedad, Planta y Equipo, del sistema electríco del cantón La Troncal de propiedad de la CNEL EP REGIONAL MILAGRO, según valores históricos.

\begin{tabular}{|c|l|r|r|c|}
\hline No. & \multicolumn{1}{|c|}{ Elemento } & $\begin{array}{c}\text { Valor } \\
\text { histórico }\end{array}$ & $\begin{array}{c}\text { Valor por } \\
\text { desmantelamiento }\end{array}$ & $\%$ \\
\hline $\mathbf{1}$ & $\begin{array}{l}\text { Edificios y estructuras } \\
\text { subestación la troncal }\end{array}$ & $36.175,20$ & $2.961,59$ & 8,19 \\
\hline $\mathbf{2}$ & $\begin{array}{l}\text { Equipos de subestación la } \\
\text { troncal }\end{array}$ & $359.803,19$ & $29.456,33$ & 8,19 \\
\hline
\end{tabular}




\begin{tabular}{|c|c|c|c|c|}
\hline 3 & $\begin{array}{l}\text { Postes, torres y accesorios } \\
\text { línea de s/t s/e troncal- s/e } \\
\text { el triunfo }\end{array}$ & $469.946,80$ & $38.473,56$ & 8,19 \\
\hline 4 & $\begin{array}{l}\text { Conductores y accesorios } \\
\text { en línea de s/t s/e troncal - } \\
\text { s/e el triunfo }\end{array}$ & $182.613,04$ & $14.950,15$ & 8,19 \\
\hline 5 & $\begin{array}{l}\text { Postes, torres y accesorios } \\
\text { en M.T. y B.T. la troncal }\end{array}$ & 2.432.191,88 & $302.175,49$ & 12,42 \\
\hline 6 & $\begin{array}{l}\text { Conductores y accesorios } \\
\text { en M.T. y B.T. la troncal }\end{array}$ & $1.630 .109,35$ & $202.524,76$ & 12,42 \\
\hline 7 & $\begin{array}{l}\text { Transformadores de } \\
\text { distribución la troncal }\end{array}$ & $1.212 .367,68$ & $150.624,54$ & 12,42 \\
\hline 8 & $\begin{array}{l}\text { Equipos de protección y } \\
\text { seccionamiento }\end{array}$ & $48.791,63$ & $6.061,87$ & 12,42 \\
\hline 9 & $\begin{array}{l}\text { Sistema de alumbrado } \\
\text { público la troncal }\end{array}$ & $1.037 .549,47$ & $296.869,55$ & 28,61 \\
\hline 10 & $\begin{array}{l}\text { Acometidas en sistemas de } \\
\text { medición la troncal }\end{array}$ & $388.297,12$ & $111.101,78$ & 28,61 \\
\hline \multirow[t]{2}{*}{11} & $\begin{array}{l}\text { Medidores de energía la } \\
\text { troncal }\end{array}$ & $626.826,14$ & $179.351,06$ & 28,61 \\
\hline & $\begin{array}{l}\text { Total general por } \\
\text { desmantelamiento }\end{array}$ & $8.424 .671,50$ & $1.334 .550,68$ & 15,84 \\
\hline
\end{tabular}

Fuente: Elaboración propia a partir de los datos validados por el Perito

El valor total por desmantelamiento del Sistema Eléctrico del Cantón la Troncal de propiedad de la de la CENTROSUR C.A, asciende a 570.627,19 USD, que representa el $17,26 \%$ en promedio, con respecto al valor total de los activos históricos, dicho porcentaje difiere para cada una de las etapas funcionales de acuerdo a la vida útil de los activos; para aquellos que tienen una vida útil de 30 años el porcentaje del desmantelamiento es del 8,19\%, para los activos con una vida útil de 25 años es el $12,42 \%$ y para los activos que tienen una vida útil de 15 años es del 28,61\%; no obstante, en valores absolutos no son iguales, debido a que los valores históricos son diferentes, como se detalla en la Tabla 3.

Tabla 3. Resumen de valores totales por desmantelamiento de Propiedad, Planta y Equipo de la CENTROSUR C.A, según valores historicos

\begin{tabular}{|c|l|c|c|c|}
\hline No. & \multicolumn{1}{|c|}{ Elemento } & Valor histórico & $\begin{array}{c}\text { Valor por } \\
\text { desmantelamiento }\end{array}$ & \% \\
\hline $\mathbf{1}$ & $\begin{array}{l}\text { Edificios y estructuras subestación La } \\
\text { Troncal }\end{array}$ & $16.422,51$ & $1.344,48$ & 8,19 \\
\hline $\mathbf{2}$ & Equipos de subestación La Troncal & $204.059,55$ & $16.705,93$ & 8,19 \\
\hline $\mathbf{3}$ & $\begin{array}{l}\text { Postes, torres y accesorios línea de } \\
\text { s/t s/e Troncal - s/e El Triunfo }\end{array}$ & & & \\
\hline $\mathbf{4}$ & $\begin{array}{l}\text { Conductores y accesorios en linea de } \\
\text { s/t s/e Troncal - s/e El Triunfo }\end{array}$ & & & \\
\hline
\end{tabular}




\begin{tabular}{|c|c|c|c|c|}
\hline No & Elemento & Valor histórico & $\begin{array}{c}\text { Valor por } \\
\text { desmantelamiento }\end{array}$ & $\%$ \\
\hline 5 & $\begin{array}{l}\text { Postes, torres y accesorios en M.T. y } \\
\text { B.T. La Troncal }\end{array}$ & $1.196 .890,99$ & $148.701,72$ & 12,42 \\
\hline 6 & $\begin{array}{l}\text { Conductores y accesorios en M.T. y } \\
\text { B.T. La Troncal }\end{array}$ & $331.027,51$ & $41.126,85$ & 12,42 \\
\hline 7 & $\begin{array}{l}\text { Transformadores de distribución La } \\
\text { Troncal }\end{array}$ & $468.970,97$ & $58.264,95$ & 12,42 \\
\hline 8 & $\begin{array}{l}\text { Equipos de protección y } \\
\text { seccionamiento }\end{array}$ & $42.441,68$ & $5.272,95$ & 12,42 \\
\hline 9 & $\begin{array}{l}\text { Sistema de alumbrado público La } \\
\text { Troncal }\end{array}$ & $366.586,14$ & $104.889,71$ & 28,61 \\
\hline 10 & $\begin{array}{l}\text { Acometidas en sistemas de medición } \\
\text { La Troncal }\end{array}$ & $243.966,36$ & $69.805,04$ & 28,61 \\
\hline 11 & Medidores de energía La Troncal & $435.177,86$ & $124.515,56$ & 28,61 \\
\hline & Total general por desmantelamiento & $3.305 .543,57$ & $570.627,19$ & 17,26 \\
\hline
\end{tabular}

Fuente: Elaboración propia a partir de los datos validados por el Perito.

Se presenta el resumen comparativo del efecto del cálculo del desmantelamiento de la Propiedad, Planta y Equipo del sistema eléctrico de la CNEL EP REGIONAL MILAGRO y de la CENTROSUS C.A., con sus respectivos valores, el mismo que representa en total 1905.177,87 USD, como se detalla en la Tabla 3.

Tabla 4. Valor total por desmantelamiento de Propiedades, Planta y Equipo del sistema electríco del cantón La Troncal, de propiedad de la CNEL EP REGIONAL MILAGRO Y DE LA CENTROSUR C.A, según valores históricos.

\begin{tabular}{|c|l|c|c|c|c|}
\hline & & \multicolumn{2}{|c|}{ CNEL EP } & \multicolumn{2}{c|}{ CENTROSUR C.A } \\
\hline $\mathbf{1}$ & \multicolumn{1}{|c|}{ Elemento } & Valor histórico & $\begin{array}{l}\text { Valor por desa- } \\
\text { mantelamiento }\end{array}$ & Valor histórico & $\begin{array}{l}\text { Valor por desman- } \\
\text { telamiento }\end{array}$ \\
\hline $\mathbf{2}$ & $\begin{array}{l}\text { Edificios y estructu- } \\
\text { ras subestación La } \\
\text { Troncal }\end{array}$ & $36.175,20$ & $2.961,59$ & $16.422,51$ & $1.344,48$ \\
\hline $\mathbf{3}$ & $\begin{array}{l}\text { Equipos de subesta- } \\
\text { ción La Troncal }\end{array}$ & $359.803,19$ & $29.456,33$ & $204.059,55$ & $16.705,93$ \\
\hline $\mathbf{4}$ & $\begin{array}{l}\text { Postes, torres y ac- } \\
\text { sesorios línea de } \\
\text { Triunfo Troncal-s/e El }\end{array}$ & $469.946,80$ & $38.473,56$ & & \\
\hline $\begin{array}{l}\text { Conductores y acce- } \\
\text { sorios en línea de s/t } \\
\text { s/e Troncal - s/e El } \\
\text { Triunfo }\end{array}$ & $182.613,04$ & $14.950,15$ & $148.701,72$ \\
\hline $\mathbf{5}$ & $\begin{array}{l}\text { Postes, torres y acce- } \\
\text { sorios en M.T. y B.T. } \\
\text { la troncal }\end{array}$ & $2.432 .191,88$ & $302.175,49$ & $1.196 .890,99$ & \\
\hline
\end{tabular}




\begin{tabular}{|c|l|c|c|c|c|}
\hline & & \multicolumn{2}{|c|}{ CNEL EP } & \multicolumn{2}{|c|}{ CENTROSUR C.A } \\
\hline No. & \multicolumn{1}{|c|}{ Elemento } & Valor histórico & $\begin{array}{l}\text { Valor por desa- } \\
\text { mantelamiento }\end{array}$ & Valor Histórico & $\begin{array}{c}\text { Valor por desman- } \\
\text { telamiento }\end{array}$ \\
$\mathbf{6}$ & $\begin{array}{l}\text { Conductores y acce- } \\
\text { sorios en M.T. y B.T. } \\
\text { la troncal }\end{array}$ & $1.630 .109,35$ & $202.524,76$ & $331.027,51$ & $41.126,85$ \\
\hline $\mathbf{7}$ & $\begin{array}{l}\text { Transformadores de } \\
\text { distribución la troncal }\end{array}$ & $1.212 .367,68$ & $150.624,54$ & $468.970,97$ & $58.264,95$ \\
\hline $\mathbf{8}$ & $\begin{array}{l}\text { Equipos de protec- } \\
\text { ción y seccionamien- } \\
\text { to }\end{array}$ & $48.791,63$ & $6.061,87$ & $42.441,68$ & $5.272,95$ \\
\hline $\mathbf{9}$ & $\begin{array}{l}\text { Sistema de alumbra- } \\
\text { do público La Troncal }\end{array}$ & $1.037 .549,47$ & $296.869,55$ & $366.586,14$ & $104.889,71$ \\
\hline $\mathbf{1 0}$ & $\begin{array}{l}\text { Acometidas en siste- } \\
\text { mas de medición La } \\
\text { Troncal }\end{array}$ & $388.297,12$ & $111.101,78$ & $243.966,36$ & $69.805,04$ \\
\hline $\mathbf{1 1}$ & $\begin{array}{l}\text { Medidores de energía } \\
\text { La Troncal }\end{array}$ & $\mathbf{6 2 6 . 8 2 6 , 1 4}$ & $179.351,06$ & $435.177,86$ & $124.515,56$ \\
\hline & \multicolumn{1}{|c|}{ TOTAL USD } & $\mathbf{8 . 4 2 4 . 6 7 1 , 5 0}$ & $\mathbf{1 . 3 3 4 . 5 5 0 , 6 8}$ & $\mathbf{3 . 3 0 5 . 5 4 3 , 5 7}$ & $\mathbf{5 7 0 . 6 2 7 , 1 9}$ \\
\hline
\end{tabular}

Fuente: Elaboración propia a partir de los datos validados por el Perito.

En la Figura 1, se demuestra los valores totales por concepto del desmantelamiento que se aplicaría en los registros contables de Propiedades, Planta y Equipo de la CNEL EP REGIONAL MILAGRO Y DE LA CENTROSUR C.A, según valores históricos, con el respectivo impacto en los activos totales.

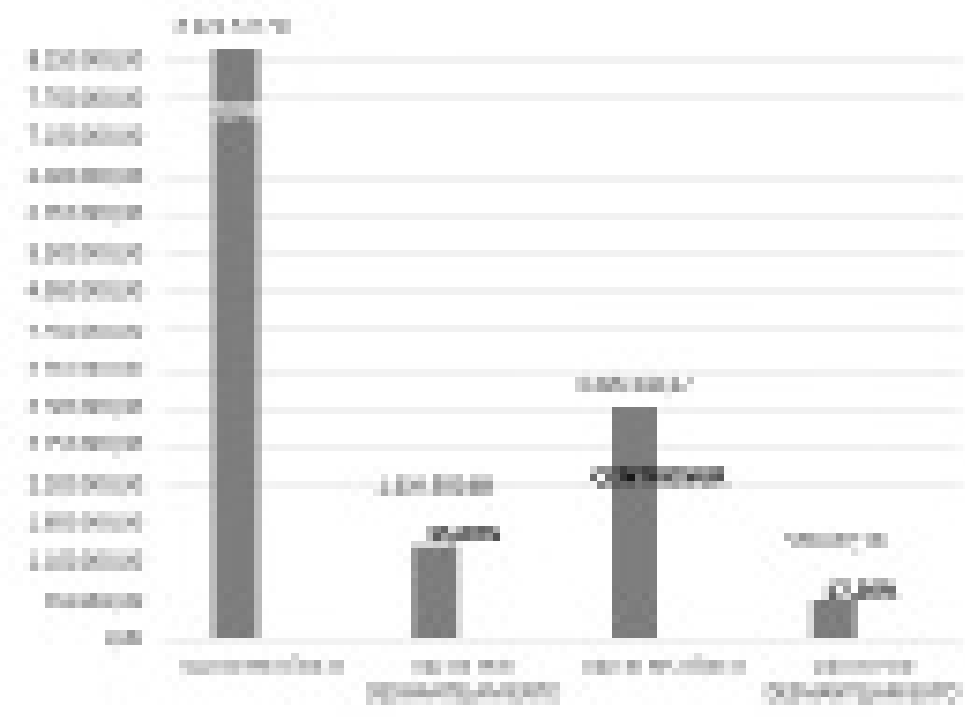

Figura 1. Valores históricos y costos por desmantelamiento de Propiedad, Planta y Equipo del sistema eléctrico de la CNEL EP y de la CENTROSUR C.A.

El resultado final del impacto financiero es un incremento en el activo por el nuevo costo por desmantelamiento, incremento en el pasivo por efecto de la cuenta denominada provisión por desmantelamiento y el incremento en las cuentas de gastos de provisión por desmantelamiento y el gasto financiero por desmantelamiento, 
lo que significa finalmente un incremento de los gastos con afectación directa a los resultados finales, esto es, una disminución del superávit o incremento del déficit.

El efecto final por el incremento en el valor de los activos por DESMANTELAMIENTO DE PROPIEDADES, PLANTA Y EQUIPO DE LA CNEL EP, representa el 3\% con respecto al costo histórico y el $13 \%$ por gasto financiero, que se acreditará paulatinamente, durante toda la vida útil del activo; en el caso de la CENTROSUR C.A el total general del desmantelamiento representa en promedio el $4 \%$ con respecto al costo histórico y el $14 \%$ por gasto financiero durante toda la vida útil del activo, como se detalla en las Tablas 5 y 6.

Tabla 5. Incremento en el valor de los activos por desmantelamiento y gasto financiero de Propiedades, Planta y Equipo del Sistema Eléctrico del Cantón La Troncal, de propiedad de la CNEL EP REGIONAL MILAGRO

\begin{tabular}{|c|c|c|c|c|c|}
\hline No & Elemento & $\begin{array}{c}\text { Valor histórico } \\
\text { del activo }\end{array}$ & $\begin{array}{l}\text { Incremento del acti- } \\
\text { vo por desmantela- } \\
\text { miento }\end{array}$ & $\begin{array}{l}\text { Gasto finan- } \\
\text { ciero }\end{array}$ & $\begin{array}{l}\text { Valor del desmante- } \\
\text { lameinto }\end{array}$ \\
\hline 1 & $\begin{array}{l}\text { Edificios y es- } \\
\text { tructuras }\end{array}$ & $36.175,20$ & 242,46 & $2.719,13$ & $2.961,59$ \\
\hline 2 & $\begin{array}{l}\text { Equipos de } \\
\text { subestación }\end{array}$ & $359.803,19$ & $2.411,53$ & $27.044,81$ & $29.456,33$ \\
\hline 3 & $\begin{array}{l}\text { Postes, torres } \\
\text { y accesorios } \\
\text { línea de } s / t\end{array}$ & $469.946,80$ & $3.149,75$ & $35.323,81$ & $38.473,56$ \\
\hline 4 & $\begin{array}{l}\text { Conductores y } \\
\text { accesorios en } \\
\text { línea de s/t }\end{array}$ & $182.613,04$ & $1.223,94$ & $13.726,21$ & $14.950,15$ \\
\hline 5 & $\begin{array}{l}\text { Postes, torres y } \\
\text { accesorios en } \\
\text { m.t. y b.t. }\end{array}$ & 2.432.191,88 & $37.542,28$ & $264.633,21$ & $302.175,49$ \\
\hline 6 & $\begin{array}{l}\text { Conductores y } \\
\text { accesorios en } \\
\text { m.t. y b.t. }\end{array}$ & $1.630 .109,35$ & $25.161,67$ & $177.363,09$ & $202.524,76$ \\
\hline 7 & $\begin{array}{l}\text { Transformado- } \\
\text { res de distribu- } \\
\text { ción }\end{array}$ & $1.212 .367,68$ & $18.713,59$ & $131.910,95$ & $150.624,54$ \\
\hline 8 & $\begin{array}{l}\text { Equipos de } \\
\text { protección y } \\
\text { seccionamiento }\end{array}$ & $48.791,63$ & 753,13 & $5.308,74$ & $6.061,87$ \\
\hline 9 & $\begin{array}{l}\text { Sistema de } \\
\text { alumbrado } \\
\text { público }\end{array}$ & $1.037 .549,47$ & $84.942,00$ & $211.927,55$ & $296.869,55$ \\
\hline 10 & $\begin{array}{l}\text { Acometidas en } \\
\text { sistemas de } \\
\text { medición }\end{array}$ & $388.297,12$ & $31.789,07$ & $79.312,71$ & $111.101,78$ \\
\hline 11 & $\begin{array}{l}\text { Medidores de } \\
\text { energía }\end{array}$ & $626.826,14$ & $51.316,94$ & $128.034,11$ & $179.351,06$ \\
\hline & TOTAL & $8.424 .671,50$ & $257.246,36$ & $1.077 .304,32$ & $1.334 .550,68$ \\
\hline & & $100 \%$ & $3 \%$ & $13 \%$ & \\
\hline
\end{tabular}

Fuente: Elaboración propia a partir de los datos validados por el Perito. 
Tabla 5. Incremento en el valor de los activos por desmantelamiento y gasto financiero de Propiedades, Planta y Equipo del Sistema Eléctrico de la Centrosur C.A.

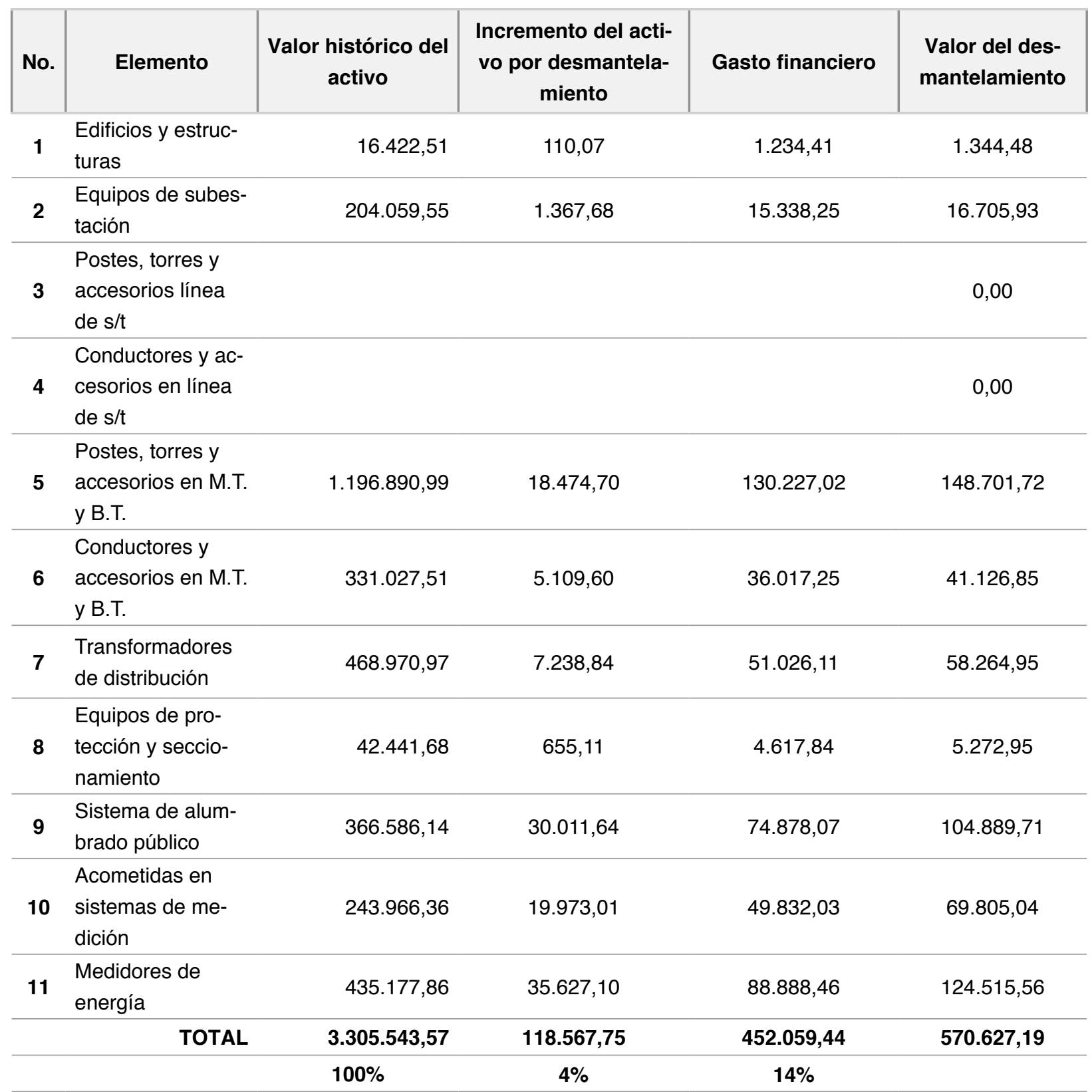

Fuente: Elaboración propia a partir de los datos validados por el Perito.

Finalmente, el impacto total en la Propiedades, Planta y Equipo de la CNEL EP REGIONAL MILAGRO Y DE LA CENTROSUR C.A, representa el $3 \%$ respecto al incremento de los activos y el $13 \%$ por gasto financiero, como se demuestra en la Figura 2. 


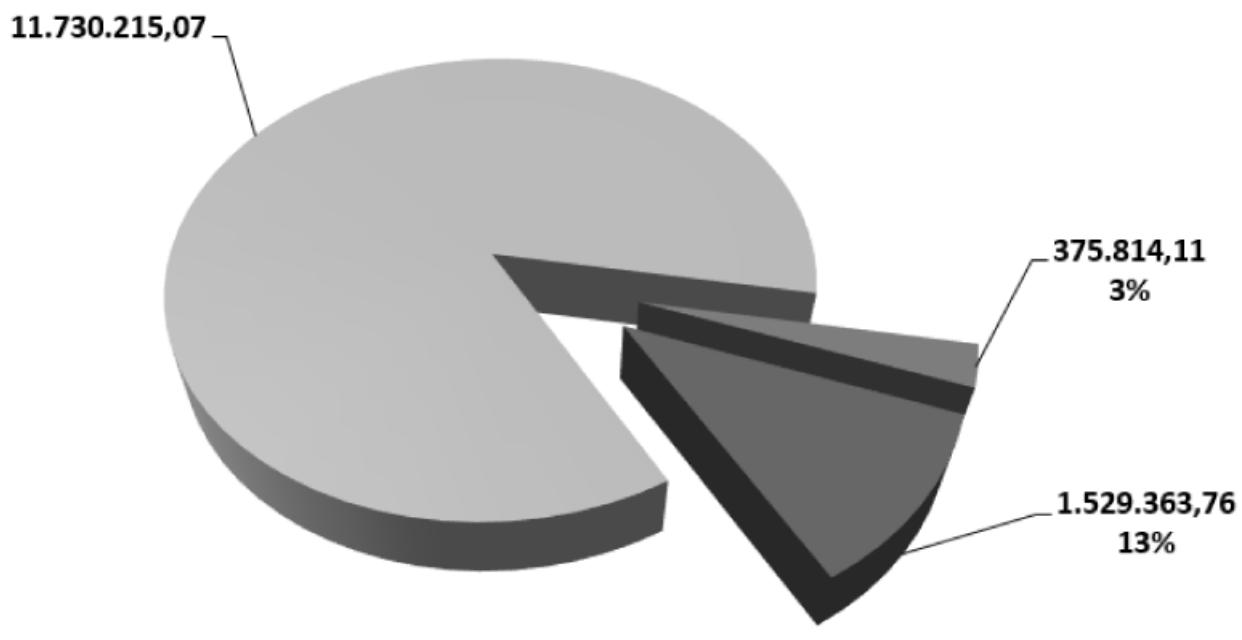

- VALOR HISTÓRICO DEL ACTIVO

Figura 2. Valor de los activos de propiedad de la CNEL EP regional Milagro y de la CENTROSUR CA, según el incremento por desmantelamiento y los gastos financieros.

El efecto final es un incremento en el registro contable de las Propiedades, Planta y Equipo por el valor de 375.814,11 USD, lo que mejora la situación financiera de la CNEL EP regional Milagro y de la CENTROSUR CA.; sin embargo, durante la vida útil estimada de los activos, se tendrá que cargar a los gastos por depreciación, gasto por provisión y gasto financiero por desmantelamiento el valor total de 1 529.363,76 USD; afectando a la situación económica y disminuyendo el superávit o incrementando el déficit, de acuerdo a los resultados finales que obtenga las empresas del sistema eléctrico por cada año, durante la vida útil estimada para las Propiedades, Planta y Equipo.

\section{5.- Conclusiones}

- En los informes de los Auditores Externos y Comisario constan recomendaciones referentes a la necesidad de realizar un estudio sobre las reservas para el desmantelamiento y restauración del medio ambiente para los activos de la empresa, de conformidad a lo dispuesto por las Normas Internacionales de Contabilidad 16 y 37.

- La estimación del valor para el desmantelamiento se realizó considerando el valor total del costo histórico de los activos valorados, clasificados por sus etapas funcionales, al cual se aplica el cálculo del valor actual como costo alternativo del capital que se invertirá, para obtener el valor presente de una cantidad que se espera erogar en algún momento del futuro, llegando al valor total de 1 905.177,87 USD, desagregado en $1334.550,68$ USD para la CNEL EP y 570.627,19 USD para la CENTROSUR C.A. Valores que se destinarán para la fase de retiro, que incluye la parte técnica (desarmado; derrocamiento y producción de escombros; transporte de piezas y escombros); y, la parte ambiental (disposición final de residuos y desechos; conformación de suelo y cobertura vegetal, entre otros); esto es, el costo ambiental de remediación que se pueda incurrir al momento de retirar el activo. 
- El valor total de reemplazo a nuevo asciende a $11730.215,09$ USD; de este valor se obtiene el $20,11 \%$ que representa el costo de la mano de obra para el montaje del activo, definido en función de los precios establecidos por la CENTROSUR C.A, que representa el valor de 2 358.946,23 USD; y, el porcentaje estimado para el valor de la mano de obra para el desmontaje es de aproximadamente el $60 \%$ del costo de la mano de obra para el montaje, que asciende a $1415.367,74$ USD. Este valor no incluye el costo ambiental para remediación para retiro del activo, con lo cual se demuestra que el valor determinado para el desmantelamiento, esto es, de 1905.177,87 USD, es un monto calculado con un procedimiento metodológico y técnico muy cercano a la realidad y que no causará desajustes drásticos en la situación económica y financiera de la empresa.

- El servidor responsable del registro contable, debe informar mediante notas explicativas a los estados financieros sobre el incremento del activo, pasivo contingente, incremento de los gastos y disminución del superávit o incremento del déficit, producto de la aplicación de las Normas Internacionales de Contabilidad 16 y 37 , referente al desmantelamiento.

- Se puede incrementar o disminuir el valor del desmantelamiento por la variación de las tasas de inflación, o por la fluctuación de la tasa de interés activa referencial que emite periódicamente el Banco Central del Ecuador; o, por cualquier otro parámetro que finalmente la empresa pueda decidir para provisionar los valores por desmantelamiento.

- Todas las empresas y entidades deben aplicar las NIC 16 y 37, respecto al desmantelamiento y provisión del pasivo, con la finalidad de presentar estados financieros apegados a la realidad, para la toma de decisiones oportunas. 


\section{Referencias}

- IFRS FUNDATION (2017), NIIF Pymes

- Servicio de Rentas Internas SRI (2010) Reglamento a la Ley Orgánica de Régimen Tributario Interno. Registro Oficial Suplemento 209 del 8 de junio de 2010, Decreto Ejucutivo No. 374

- Mantilla, S.A. (2012). Estándares/Normas Internacionales de Información Financiera IFRS (NIIF) (Tercera edición) Bogotá: Ecoe Ediciones.

- Ministerio de Finanzas, Acuerdo ministerial 037 (2012). Ecuador

- IICE y FNCE (2009). Plan de adopción y socialización en el Ecuador de las Normas Internacionales de Información Financiera y de las Normas Internacionales de Auditoría y Aseguramiento. Guayaquil.

- Superintendencia de Compañías del Ecuador (2018), Portal de Información.

- Mundial,B. (2004), Informe Sobre la Observancia de los Códigos y las Normas.

- Instituto Ecuatoriano de Electrificación (INECEL) 1961.

- Rodríguez, J., (2014), valoración, confirmación o rectificación de la valoración de la Propiedades, Planta y Equipos (Activos Fijos), incluye estimación por desmantelamiento, de propiedad de la empresa eléctrica regional Centro Sur, con corte a junio de 2016. 